\title{
A DUALITY THEOREM FOR INTERPOLATION METHODS ASSOCIATED TO POLYGONS
}

\author{
FERNANDO COBOS AND PEDRO FERNANDEZ-MARTINEZ
}

(Communicated by Palle E. T. Jorgensen)

\begin{abstract}
We investigate dual spaces of interpolation spaces defined by means of polygons. We first show that dual spaces may fail to be intermediate spaces with respect to the dual $N$-tuple, and then we prove that dual spaces of $J$ spaces can be identified with closed subspaces of $K$-spaces generated by the dual $N$-tuple.
\end{abstract}

\section{INTRODUCTION}

Duality is one of the points in interpolation theory where one meets serious obstructions in extending results on classical interpolation methods for couples of Banach spaces to methods defined with respect to an $N$-tuple $(N \geq 3)$. For instance, the well-known equality for couples

$$
\Sigma(\bar{A})^{\prime}=\Delta\left(\overline{A^{\prime}}\right)
$$

(see [1, Theorem 2.7.1]) is no longer valid for an $N$-tuple of Banach spaces (see [9]). This setback means that duals of interpolation spaces generated by an $N$-tuple might not be intermediate spaces with respect to the dual $N$-tuple .

Duality for interpolation methods that extend the classical complex method to $N$-tuples has been discussed in a number of papers in the literature (see, for example, $[6,14,10,8]$ ), but it seems that very few results are known on duality for multidimensional methods related to the classical real method. The aim of the present note is to investigate this question.

We establish a duality result for interpolation methods defined by means of polygons $\Pi$ in the plane $\mathbb{R}^{2}$ (see [5]). In particular, our result applies to Sparr's spaces [15] (case when $\Pi$ is equal to the simplex) and to Fernandez's spaces [11] ( $\Pi$ equal to the unit square). For the latter, this problem was studied earlier by Fernandez [12], but his arguments have some inaccuracies (see [13]). In fact, our results show that certain duality formulae in [12] are incorrect.

Received by the editors November 3, 1992.

1991 Mathematics Subject Classification. Primary 46B70, 46M35.

The first author was supported in part by US-Spain Joint Committee for Cultural and Educational Cooperation (Grant II-C 91024). 


\section{INTERPOLATION METHODS DEFINED BY MEANS OF POLYGONS}

Let $\bar{A}=\left\{A_{1}, \ldots, A_{N}\right\}$ be a Banach $N$-tuple, that is, $N$ Banach spaces $A_{j} \quad(1 \leq j \leq N)$ which are continuously embedded in a common Hausdorff topological vector space. Their intersection $\Delta(\bar{A})=A_{1} \cap \cdots \cap A_{N}$ and their sum $\Sigma(\bar{A})=A_{1}+\cdots+A_{N}$ are then also Banach spaces when normed by

$$
\|a\|_{\Delta(\bar{A})}=\max _{1 \leq j \leq N}\left\{\|a\|_{A_{j}}\right\}
$$

and

$$
\|a\|_{\Sigma(\bar{A})}=\inf \left\{\sum_{j=1}^{N}\left\|a_{j}\right\|_{A_{j}}: a=\sum_{j=1}^{N} a_{j}, \quad a_{j} \in A_{j}\right\} .
$$

Let $\Pi=\overline{P_{1} \cdots P_{N}}$ be a convex polygon in the plane $\mathbb{R}^{2}$, with vertices $P_{j}=$ $\left(x_{j}, y_{j}\right)$. In what follows, it will be useful to imagine each space $A_{j}$ from the $N$-tuple $\bar{A}$ as sitting on the vertex $P_{j}(j=1, \ldots, N)$.

Given any two positive numbers $t, s$, we may equivalently renorm $\Delta(\bar{A})$ by the $J$-functional (with respect to the polygon $\Pi$ )

$$
J(t, s ; a)=\max _{1 \leq j \leq N}\left\{t^{x_{j}} s^{y_{j}}\|a\|_{A_{j}}\right\} .
$$

Similarly, the $K$-functional

$$
K(t, s ; a)=\inf \left\{\sum_{j=1}^{N} t^{x_{j}} s^{y_{j}}\left\|a_{j}\right\|_{A_{j}}: a=\sum_{j=1}^{N} a_{j}, a_{j} \in A_{j}\right\}
$$

defines an equivalent norm to \|\|$_{\Sigma(\bar{A})}$.

Let now $1 \leq q \leq \infty$, and let $(\alpha, \beta)$ be any interior point of $\Pi[(\alpha, \beta) \in$ Int $\Pi$ ]. The interpolation space $\bar{A}_{(\alpha, \beta), q ; K}$ is defined as the set of all elements $a \in \Sigma(\bar{A})$ having finite norm

$$
\|a\|_{(\alpha, \beta), q ; K}=\left(\sum_{(m, n) \in \mathbb{Z}^{2}}\left(2^{-\alpha m-\beta n} K\left(2^{m}, 2^{n} ; a\right)\right)^{q}\right)^{1 / q} .
$$

On the other hand, the $J$-space $\bar{A}_{(\alpha, \beta), q ; J}$ consists of all those elements $a \in$ $\Sigma(\bar{A})$ which can be represented in the form

$$
a=\sum_{(m, n) \in \mathbb{Z}^{2}} u_{m, n}
$$

(convergence in $\Sigma(\bar{A})$ ) with $\left(u_{m, n}\right) \subset \Delta(\bar{A})$ and

$$
\left(\sum_{(m, n) \in \mathbb{Z}^{2}}\left(2^{-\alpha m-\beta n} J\left(2^{m}, 2^{n} ; u_{m, n}\right)\right)^{q}\right)^{1 / q}<\infty .
$$


We equip this space with the norm

$$
\|a\|_{(\alpha, \beta), q ; J}=\inf \left\{\left(\sum_{(m, n) \in \mathbb{Z}^{2}}\left(2^{-\alpha m-\beta n} J\left(2^{m}, 2^{n} ; u_{m, n}\right)\right)^{q}\right)^{1 / q}\right\}
$$

where the infimum is taken over all representations $\left(u_{m, n}\right)$ as above.

These interpolation spaces were introduced by Peetre and the first author in [5]. Besides this article, we also refer to [4, 2, 3] for the theory of interpolation spaces associated to polygons.

It is possible to give a continuous characterization of $\bar{A}_{(\alpha, \beta), q ; K}$ and $\bar{A}_{(\alpha, \beta), q ; J}$, using integrals instead of sums, but it will not be required here. In contrast to the classical case of Banach couples, where $K$ - and $J$-spaces coincide to within equivalence of norms, in our multidimensional context we only have that $\bar{A}_{(\alpha, \beta), q ; J}$ is continuously embedded in $\bar{A}_{(\alpha, \beta), q ; K}$. They are different in general.

Next we show some concrete cases of these spaces.

Example 1.1. If $\Pi$ is equal to the simplex $\{(0,0),(1,0),(0,1)\}$ and $\alpha>$ $0, \beta>0$ with $\alpha+\beta<1$, we recover spaces studied by Sparr in [15].

Example 1.2. If $\Pi$ coincides with the unit square $\{(0,0),(1,0),(0,1),(1,1)\}$ and $0<\alpha, \beta<1$, then we obtain spaces considered by Fernandez in $[11,12$, 13].

The following estimate will be crucial for the counterexample in $\S 2$, and has independent interest. For $a \in \Delta(\bar{A})$, it relates its norm $\|a\|_{(\alpha, \beta), q ; J}$ in $\bar{A}_{(\alpha, \beta), q ; J}$ to norms in the $A_{j}$ 's.

Given $(\alpha, \beta) \in$ Int $\Pi$ where $\Pi=\overline{P_{1} \cdots P_{N}}$, we denote by $\mathscr{P}_{\alpha, \beta}$ the collection of all those triples $\{i, k, r\}$ such that $(\alpha, \beta)$ belongs to the triangle with vertices $P_{i}, P_{k}, P_{r}$.

Theorem 1.3. Let $\Pi=\overline{P_{1} \cdots P_{N}}$ be a convex polygon with $P_{j}=\left(x_{j}, y_{j}\right)$ for $j=$ $1, \ldots, N$, let $1 \leq q \leq \infty,(\alpha, \beta) \in \operatorname{Int} \Pi$, and let $\mathscr{P}_{\alpha, \beta}$ be the set defined before. Assume that $\bar{A}=\left\{A_{1}, \ldots, A_{N}\right\}$ is a Banach $N$-tuple. Then there exists a constant $C>0$, depending only on $\Pi$ and $(\alpha, \beta)$, such that for any $a \in \Delta(\bar{A})$ we have

$$
\|a\|_{(\alpha, \beta), q ; J} \leq C \max \left\{\|a\|_{A_{i}}^{c_{i}}\|a\|_{A_{k}}^{c_{k}}\|a\|_{A_{r}}^{c_{r}}:\{i, k, r\} \in \mathscr{P}_{\alpha, \beta}\right\}
$$

where $\left(c_{i}, c_{k}, c_{r}\right)$ stands for the barycentric coordinates of $(\alpha, \beta)$ with respect to $P_{i}, P_{k}, P_{r}$.

Proof. We may assume that $q=1$, because $\bar{A}_{(\alpha, \beta), 1 ; J}$ is continuously embedded in $\bar{A}_{(\alpha, \beta), q ; J}$ with norm 1 .

Given any $a \in \Delta(\bar{A})$ and $(m, n) \in \mathbb{Z}^{2}$, the sequence

$$
u_{r, s}= \begin{cases}a & \text { if }(r, s)=(m, n) \\ 0 & \text { otherwise }\end{cases}
$$


is a representation of $a$ as required in the $J$-space. Therefore,

$$
\begin{aligned}
\|a\|_{(\alpha, \beta), 1 ; J} & \leq 2^{-\alpha m-\beta n} J\left(2^{m}, 2^{n} ; a\right) \\
& =\max _{1 \leq j \leq N}\left\{2^{\left(x_{j}-\alpha\right) m+\left(y_{j}-\beta\right) n}\|a\|_{A_{j}}\right\} .
\end{aligned}
$$

Since $(m, n)$ was taken arbitrarily in $\mathbb{Z}^{2}$, we obtain that

$$
\begin{aligned}
\|a\|_{(\alpha, \beta), 1 ; J} & \leq \inf _{(m, n) \in \mathbb{Z}^{2}}\left[\max _{1 \leq j \leq N}\left\{2^{\left(x_{j}-\alpha\right) m+\left(y_{j}-\beta\right) n}\|a\|_{A_{j}}\right\}\right] \\
& \leq C \inf _{t>0, s>0}\left[\max _{1 \leq j \leq N}\left\{t^{x_{j}-\alpha} s^{y_{j}-\beta}\|a\|_{A_{j}}\right\}\right]
\end{aligned}
$$

where the constant $C$ only depends on $\Pi$ and $(\alpha, \beta)$. The last infimum was calculated in [3, Theorem 1.9]. It turned out to be

$$
\max \left\{\|a\|_{A_{i}}^{c_{i}}\|a\|_{A_{k}}^{c_{k}}\|a\|_{A_{r}}^{c_{r}}:\{i, k, r\} \in \mathscr{P}_{\alpha, \beta}\right\}
$$

where $0 \leq c_{i}, c_{k}, c_{r} \leq 1, c_{i}+c_{k}+c_{r}=1$, and $(\alpha, \beta)=c_{i} P_{i}+c_{k} P_{k}+c_{r} P_{r}$. This proves the assertion.

\section{Duality}

Subsequently, we suppose that the Banach $N$-tuple $\bar{A}=\left\{A_{1}, \ldots, A_{N}\right\}$ is regular, meaning that $\Delta(\bar{A})$ is dense in each $A_{j}(j=1, \ldots, N)$. Then the dual space $A_{j}^{*}$ of $A_{j}$ can be identified with a subspace $A_{j}^{\prime}$ of $\Delta(\bar{A})^{*}$. Thus, the family $\overline{A^{\prime}}=\left\{A_{1}^{\prime}, \ldots, A_{N}^{\prime}\right\}$ is a Banach $N$-tuple too. Moreover, according to [9, Proposition 4.1], we have that

$$
\Delta(\bar{A})^{*}=\Sigma\left(\overline{A^{\prime}}\right) \quad \text { (equal norms). }
$$

It follows easily from the definition of the $J$-space that $\Delta(\bar{A})$ is dense in $\bar{A}_{(\alpha, \beta), q ; J}$ if $q<\infty$. Hence we can also realize the dual space of $\bar{A}_{(\alpha, \beta), q ; J}$ as a subspace $\left(\bar{A}_{(\alpha, \beta), q ; J}\right)^{\prime}$ of $\Delta(\bar{A})^{*}=\Sigma\left(\overline{A^{\prime}}\right)$. But now, unlike the case of the classical real method, the space $\left(\bar{A}_{(\alpha, \beta), q ; J}\right)^{\prime}$ may fail to be an intermediate space with respect to the dual $N$-tuple $\overline{A^{\prime}}$. Next we show this by means of a counterexample where

$$
\Delta\left(\overline{A^{\prime}}\right) \not \subset\left(\bar{A}_{(\alpha, \beta), q ; J}\right)^{\prime} .
$$

Our construction is similar to those used in [6, Appendix 1] and $[10, \S 6]$ to investigate certain questions on multidimensional complex methods.

Counterexample 2.1. Let $\Pi=\overline{P_{1} \cdots P_{N}}$ be any convex polygon with vertices $P_{j}(j=1, \ldots, N)$, and let $(\alpha, \beta) \in \operatorname{Int} \Pi$ such that $(\alpha, \beta)$ belongs to the interior of the triangles $\overline{P_{1} P_{N-1} P_{N}}$ and $\overline{P_{N-2} P_{N-1} P_{N}}$ (see Figure 2.1). 


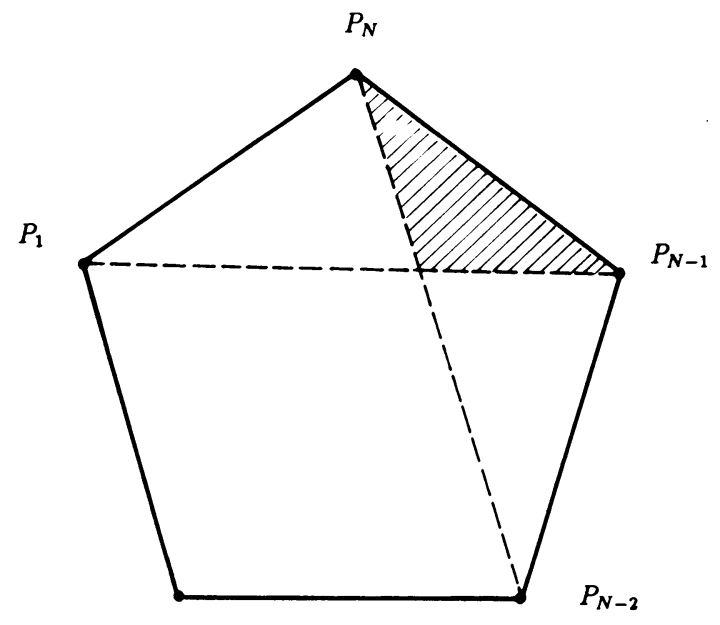

FIGURE 2.1

Consider the following Banach $N$-tuple $\bar{A}$ of scalar sequence spaces:

$$
\begin{gathered}
A_{1}=\cdots=A_{N-2}=\left\{\xi=\left(\xi_{m}\right):\|\xi\|_{\ell_{1}}=\sum_{m=-\infty}^{\infty}\left|\xi_{m}\right|<\infty \text { and } \sum_{m=-\infty}^{\infty} \xi_{m}=0\right\}, \\
A_{N-1}=\left\{\xi=\left(\xi_{m}\right):\|\xi\|_{A_{N-1}}=\sum_{m=-\infty}^{\infty} \min \left\{1,2^{m}\right\}\left|\xi_{m}\right|<\infty\right\} \\
A_{N}=\left\{\xi=\left(\xi_{m}\right):\|\xi\|_{A_{N}}=\sum_{m=-\infty}^{\infty} \min \left\{1,2^{-m}\right\}\left|\xi_{m}\right|<\infty\right\} .
\end{gathered}
$$

It is not hard to verify that $\Delta(\bar{A})=A_{1}$ is dense in each $A_{j}(j=1, \ldots, N)$. Thus $\bar{A}$ is a regular $N$-tuple.

Next let $f$ be the linear functional defined on $\Delta(\bar{A})$ by

$$
f(\xi)=\sum_{m=0}^{\infty} \xi_{m}=-\sum_{m=-1}^{-\infty} \xi_{m}
$$

It is clear that $f$ admits a continuous extension to each $A_{j}(j=1, \ldots, N)$. Hence $f \in \Delta\left(\overline{A^{\prime}}\right)$. Nevertheless $f \notin\left(\bar{A}_{(\alpha, \beta), q ; J}\right)^{\prime}$. We shall check it by showing a sequence $\left(z_{n}\right)_{n=1}^{\infty} \subset \Delta(\bar{A})$ such that

$$
\left(z_{n}\right) \text { converges to } 0 \text { in } \bar{A}_{(\alpha, \beta), q ; J}
$$

but

$$
f\left(z_{n}\right)=1 \text { for } n=1,2, \ldots .
$$

Given $m \in \mathbb{Z}$, we designate by $\delta^{(m)}$ the scalar sequence which is zero at all coordinates but the $m$ th coordinate where it is one, and for $n=1,2, \ldots$ we put $z_{n}=\delta^{(n)}-\delta^{(-n)}$. Obviously $\left(z_{n}\right) \subset \Delta(\bar{A})=A_{1}$ and $f\left(z_{n}\right)=1$ for 
$n=1,2, \ldots$ It remains to see that $\left(z_{n}\right)$ is a null sequence in $\bar{A}_{(\alpha, \beta), q ; J}$. For this purpose take $1 \leq n<m$ and let us estimate the norms of $\delta^{(n)}-\delta^{(m)}$ and of $\delta^{(-n)}-\delta^{(-m)}$ in $\overline{\bar{A}}_{(\alpha, \beta), q ; J}$. Since

$$
\left\|\delta^{(n)}-\delta^{(m)}\right\|_{A_{1}}=\cdots=\left\|\delta^{(n)}-\delta^{(m)}\right\|_{A_{N-1}}=2
$$

and

$$
\left\|\delta^{(n)}-\delta^{(m)}\right\|_{A_{N}}=2^{-n}+2^{-m}<2^{1-n},
$$

it follows from Theorem 1.3 and the choice of $(\alpha, \beta)$ that

$$
\begin{aligned}
\| \delta^{(n)} & -\delta^{(m)} \|_{(\alpha, \beta), q ; J} \\
& \leq C \max \left\{\left\|\delta^{(n)}-\delta^{(m)}\right\|_{A_{i}}^{c_{i}}\left\|\delta^{(n)}-\delta^{(m)}\right\|_{A_{k}}^{c_{k}}\left\|\delta^{(n)}-\delta^{(m)}\right\|_{A_{r}}^{c_{r}}:\{i, k, r\} \in \mathscr{P}_{\alpha, \beta}\right\} \\
& =C 2^{c_{1}} 2^{c_{2}} 2^{c_{3}(1-n)}
\end{aligned}
$$

for some $0<c_{1}, c_{2}, c_{3}<1$ with $c_{1}+c_{2}+c_{3}=1$. Hence

$$
\left\|\delta^{(n)}-\delta^{(m)}\right\|_{(\alpha, \beta), q ; J} \leq C 2^{1-\theta n}
$$

where $0<\theta=c_{3}<1$. For $\delta^{(-n)}-\delta^{(-m)}$ we have

$$
\left\|\delta^{(-n)}-\delta^{(-m)}\right\|_{A_{N}}=\left\|\delta^{(-n)}-\delta^{(-m)}\right\|_{A_{1}}=\cdots=\left\|\delta^{(-n)}-\delta^{(-m)}\right\|_{A_{N-2}}=2
$$

while

$$
\left\|\delta^{(-n)}-\delta^{(-m)}\right\|_{A_{N-1}}=2^{-n}+2^{-m}<2^{1-n} .
$$

Therefore, using again Theorem 1.3 and taking into account the choice of $(\alpha, \beta)$, we conclude that

$$
\left\|\delta^{(-n)}-\delta^{(-m)}\right\|_{(\alpha, \beta), q ; J} \leq C 2^{1-\mu n}
$$

where $0<\mu<1$.

For $(m, n) \in \mathbb{N}^{2}$ set

$$
\lambda^{(m, n)}=\left(\delta^{(n)}-\delta^{(m)}\right)-\left(\delta^{(-n)}-\delta^{(-m)}\right) .
$$

The previous estimates give

$$
\left\|\lambda^{(m, n)}\right\|_{(\alpha, \beta), q ; J} \leq C\left[2^{1-\theta \min \{m, n\}}+2^{1-\mu \min \{m, n\}}\right] .
$$

So

$$
\left\|\lambda^{(m, n)}-\lambda^{(k, n)}\right\|_{(\alpha, \beta), q ; J}=\left\|\lambda^{(m, k)}\right\|_{(\alpha, \beta), q ; J} \longrightarrow 0 \text { as } \min \{m, k\} \longrightarrow \infty .
$$

In other words, the sequence $\left(\lambda^{(m, n)}\right)_{m=1}^{\infty}$ is convergent in $\bar{A}_{(\alpha, \beta), q ; J}$. By compatibility, its limit must be the same as in

$$
\Sigma(\bar{A})=\left\{\xi=\left(\xi_{m}\right):\|\xi\|_{\Sigma(\bar{A})}=\sum_{m=-\infty}^{\infty} 2^{-|m|}\left|\xi_{m}\right|<\infty\right\},
$$

and in this last space it is clear that

$$
\left(\lambda^{(m, n)}\right) \longrightarrow \delta^{(n)}-\delta^{(-n)}=z_{n} \text { as } n \longrightarrow \infty
$$


Consequently

$$
\begin{aligned}
\left\|z_{n}\right\|_{(\alpha, \beta), q ; J} & =\lim _{m \longrightarrow \infty}\left\|\lambda^{(m, n)}\right\|_{(\alpha, \beta), q ; J} \\
& \leq C \lim _{m \longrightarrow \infty}\left[2^{1-\theta \min \{m, n\}}+2^{1-\mu \min \{m, n\}}\right] \\
& =C\left[2^{1-\theta n}+2^{1-\mu n}\right]
\end{aligned}
$$

which implies that $\left(z_{n}\right)$ converges to 0 in $\bar{A}_{(\alpha, \beta), q ; J}$.

Remark 2.2. Taking $\Pi$ equal to the unit square $\{(0,0),(1,0),(0,1),(1,1)\}$, the counterexample shows that the duality formula (2) in [12, Proposition 2.2.1] is generally incorrect.

Although $\left(\bar{A}_{(\alpha, \beta), q ; J}\right)^{\prime}$ is not an interpolation space in general, we still can characterize it in terms of a $K$-space generated with respect to the dual $N$-tuple $\overline{A^{\prime}}=\left\{A_{1}^{\prime}, \ldots, A_{N}^{\prime}\right\}$ :

Theorem 2.3. Let $\Pi=\overline{P_{1} \cdots P_{N}}$ be a convex polygon. Let $(\alpha, \beta) \in \operatorname{Int} \Pi, 1 \leq$ $q<\infty$, and $1 / q+1 / q^{\prime}=1$. Assume that $\bar{A}=\left\{A_{1}, \ldots, A_{N}\right\}$ is a regular $N$ tuple, and let $\bar{A}^{\prime}$ be the dual $N$-tuple. Then $\left(\bar{A}_{(\alpha, \beta), q ; J}\right)^{\prime}$ is a closed subspace of ${\overline{A^{\prime}}}_{(\alpha, \beta), q^{\prime} ; K}$ and the norms coincide in $\left(\bar{A}_{(\alpha, \beta), q ; J}\right)^{\prime}$.

Proof. Given $(m, n) \in \mathbb{Z}^{2}$, we denote by $G_{m, n}$ the Banach space $\Delta(\bar{A})$ endowed with the norm $J\left(2^{m}, 2^{n} ; \cdot\right)$. We put

$$
\begin{aligned}
X= & \ell_{q}\left(2^{-\alpha m-\beta n} G_{m, n}\right) \\
= & \left\{\left(u_{m, n}\right)_{(m, n) \in \mathbb{Z}^{2}}: u_{m, n} \in \Delta(\bar{A}),\right. \\
& \left.\left\|\left(u_{m, n}\right)\right\|_{X}=\left(\sum_{(m, n) \in \mathbb{Z}^{2}}\left(2^{-\alpha m-\beta n} J\left(2^{m}, 2^{n} ; u_{m, n}\right)\right)^{q}\right)^{1 / q}<\infty\right\}
\end{aligned}
$$

and

$$
\left.M=\left\{\left(u_{m, n}\right) \in X: \sum_{(m, n) \in \mathbb{Z}^{2}} u_{m, n}=0 \text { (convergence in } \Sigma(\bar{A})\right)\right\} .
$$

By the definition of $\bar{A}_{(\alpha, \beta), q ; J}$, we can identify this space with the quotient $X / M$. Hence, the classical result on the dual of a quotient space (see, for example, [7, Theorem 3.10.2]) gives that

$$
\left(\bar{A}_{(\alpha, \beta), q ; J}\right)^{*}=(X / M)^{*}=M^{\perp}
$$

where

$$
M^{\perp}=\left\{\bar{f} \in X^{*}: \bar{f}(M)=0\right\} .
$$

On the other hand, if we designate by $F_{-m,-n}$ the sum $\Sigma\left(\overline{A^{\prime}}\right)$ normed by $K\left(2^{-m}, 2^{-n} ; \cdot\right)$, then it follows from the equality $\Delta(\bar{A})^{*}=\Sigma\left(\overline{A^{\prime}}\right)$ that

$$
G_{m, n}^{*}=F_{-m,-n} \quad \text { (equal norms); }
$$


whence,

$$
X^{*}=\ell_{q^{\prime}}\left(2^{\alpha m+\beta n} G_{m, n}^{*}\right)=\ell_{q^{\prime}}\left(2^{\alpha m+\beta n} F_{-m,-n}\right) .
$$

Next note that if $\bar{f} \in M^{\perp}$ then for some $f \in \Delta(\bar{A})^{*}$ we have

$$
\bar{f}=(\ldots, f, f, f, \ldots) \text {. }
$$

Indeed, if $\bar{f}=\left(f_{m, n}\right) \in X^{*}$ is not a constant sequence, there are $(r, s),(i, j) \in$ $\mathbb{Z}^{2}$ and an element $a \in \Delta(\bar{A})$ such that $f_{r, s}(a) \neq f_{i, j}(a)$. Set

$$
\tilde{a}=\left(a_{m, n}\right) \quad \text { where } a_{m, n}= \begin{cases}a & \text { if }(m, n)=(r, s), \\ -a & \text { if }(m, n)=(i, j), \\ 0 & \text { otherwise. }\end{cases}
$$

Clearly $\tilde{a} \in M$, but

$$
\bar{f}(\tilde{a})=\sum_{(m, n) \in \mathbb{Z}^{2}} f_{m, n}\left(a_{m, n}\right)=f_{r, s}(a)-f_{i, j}(a) \neq 0 .
$$

Thus $\bar{f} \notin M^{\perp}$.

Consequently, $\left(\bar{A}_{(\alpha, \beta), q ; J}\right)^{\prime}$ coincides with a subspace of $\ell_{q^{\prime}}\left(2^{\alpha m+\beta n} F_{-m,-n}\right)$ formed by constant sequences. According to the definition of the $K$-space

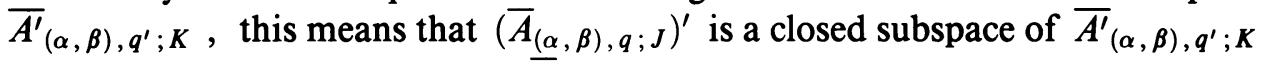
and that the norms coincide in $\left(\bar{A}_{(\alpha, \beta), q ; J}\right)^{\prime}$.

Counterexample 2.1 shows that in general

$$
\left(\bar{A}_{(\alpha, \beta), q ; J}\right)^{\prime} \neq{\overline{A^{\prime}}}_{(\alpha, \beta), q^{\prime} ; K} \text {. }
$$

\section{REFERENCES}

1. J. Bergh and J. Löfström, Interpolation spaces. An introduction, Springer, Berlin, Heidelberg, and New York, 1976.

2. F. Cobos and P. Fernández-Martinez, Reiteration and a Wolff theorem for interpolation methods defined by means of polygons, Studia Math. 102 (1992), 239-256.

3. F. Cobos, P. Fernández-Martinez, and T. Schonbek, Norm estimates for interpolation methods defined by means of polygons, J. Approx. Theory (to appear).

4. F. Cobos, T. Kühn, and T. Schonbek, One-sided compactness results for Aronszajn-Gagliardo functors, J. Funct. Anal. 106 (1992), 274-313.

5. F. Cobos and J. Peetre, Interpolation of compact operators: The multidimensional case, Proc. London Math. Soc. 63 (1991), 371-400.

6. R. R. Coifman, M. Cwikel, R. Rochberg, Y. Sagher, and G. Weiss, A theory of complex interpolation for families of Banach spaces, Adv. Math. 43 (1982), 203-229.

7. J. B. Conway, A course in functional analysis, Springer, New York, Berlin, Heidelberg, and Tokyo, 1985.

8. M. Cwikel and S. Janson, Real and complex interpolation methods for finite and infinite families of Banach spaces, Adv. Math. 66 (1987), 234-290.

9. G. Dore, D. Guidetti, and A. Venni, Some properties of the sum and intersection of normed spaces, Atti Sem. Mat. Fis. Univ. Modena 31 (1982), 325-331.

10. (1986), 1-36.

11. D. L. Fernandez, Interpolation of $2^{n}$ Banach spaces, Studia Math. 45 (1979), 175-201.

12. $\ldots$, On the duality of interpolation spaces of several Banach spaces, Acta Sci. Math. (Szeged) 44 (1982), 43-51. 
13. __ Interpolation of $2^{d}$ Banach spaces and the Calderón space $X(E)$, Proc. London Math. Soc. 56 (1988), 143-162.

14. J. Peetre, Duality for Fernandez type spaces, Math. Nachr. 119 (1984), 231-238.

15. G. Sparr, Interpolation of several Banach spaces, Ann. Mat. Pura Appl. 99 (1974), 247-316.

Departamento de Economia, Universidad Carlos III de Madrid, Calle Madrid 126, 28903 Getafe (MADRID), SPAIN

Current address, F. Cobos: Departamento de Análisis Matemático, Facultad de Matemáticas, Universidad Complutense de Madrid, 28040 Madrid, Spain

E-mail address, F. Cobos: COBOSQMAT .UCM.ES

Escuela Universitaria de INGeniería TÉcnica INdustrial de Toledo, Universidad DE Castilla-la Mancha, Avenida de Europa 28, 45005 Toledo, Spain 